\title{
A New Compounding Approach for Speckle Reduction
}

\author{
Pai-Chi Li and Mei-Ju Chen \\ Department of Electrical Engineering, National Taiwan University,
} Taipei, Taiwan, R. O. C

Abstract- A new compounding technique for reducing speckle brightness variations is proposed. This method is based on speckle decorrelation of signals under different strain states. The different strain states can be created using externally applied forces. Such forces produce 3D tissue motion. By correcting the in-plane motion, the images under different strain states have identical characteristics except for speckle appearance due to un-corrected out-of-plane motion. Therefore, speckle noise is reduced without affecting in-plane spatial resolution. Experimental results indicated that speckle brightness variations were reduced by more than $50 \%$ with an applied strain at $4.8 \%$.

\section{INTRODUCTION}

The ability of diagnostic ultrasound to detect low contrast lesions is fundamentally limited by speckle. Several compounding techniques have been proposed to reduce speckle brightness variations [1]-[4]. The improvement, however, is usually gained at the price of spatial resolution.

In this paper, a new compounding technique is proposed. This approach is based on decorrelation of images under different strain states. The different strain states can be created using externally applied forces such as the ones used in sonoelastography [5]-[6]. The external force deforms the image object producing 3D tissue motion. The in-plane motion needs to be corrected such that images under different strain conditions are properly registered and in-plane spatial resolution is not affected. The out-of-plane motion, on the other hand, is not corrected and hence the images under different strain states are partially correlated. Thus, speckle brightness variations can be reduced by incoherently averaging these partially correlated images. The proposed approach does not degrade in-plane spatial resolution since the full aperture and the entire spectral bandwidth are utilized.

The in-plane tissue motion can be estimated by two-dimensional speckle tracking. Since the proposed technique reduces speckle variations based on images under different strain states, the approach will be referred to as strain compounding.

\section{CORRELATION ANALYSIS}

There are four general steps of strain compounding: (1). Application of external forces, (2). Estimation of in-plane strain distribution, (3). Correction for in-plane tissue motion, and (4). Incoherent averaging of partially correlated measurements. If more than two measurements are available, the same procedures can be repeated and further reduction of speckle brightness variations is expected. Experimental data were acquired and analyzed using the setup shown in Fig. 1.

Gelatin based, speckle generating phantoms were constructed. The original height of the phantoms was $45 \mathrm{~mm}$. A 5 $\mathrm{MHz}$, single crystal transducer (Panametrics V310, Waltham, MA, U.S.A.) was placed on the top of the phantom. The transducer surface was flat (i.e., unfocused) with a diameter of $6 \mathrm{~mm}$. Position of the transducer was controlled by a three-axis step motor system (Q-Sync, Hsin-Chu, Taiwan, R.O.C.). Prior to data acquisition, the phantom was pre-loaded with a $4 \%$ strain and incremental compression was 
subsequently applied by moving the transducer downward with a step size of 50 $\mu \mathrm{m}$. There were totally 20 increments and the overall displacement from the pre-load position was $1 \mathrm{~mm}$. At each increment, distribution of axial displacement and correlation between signals under different compression conditions were estimated. The step motor system was also used for lateral scanning in order to construct two-dimensional B-mode images. The lateral displacement in the experiments had a $42 \mathrm{~mm}$ range with a step size of $1 \mathrm{~mm}$.

As previously mentioned, the in-plane tissue motion needs to be corrected before incoherent averaging. Without loss of generality, only axial displacement was considered and lateral displacement was ignored in our experiments.

The axial displacement was corrected by temporally stretching the RF data [6]. If the compressed phantom had a constant axial strain, the axial displacement increases linearly with the depth and thus it can be corrected by simply stretching the RF data with a factor equal to inverse of unity minus the applied strain.

The solid line in Fig. 2 shows the correlation coefficient between the original signal $x_{1}(n)$ and a stretched signal at the $i$-th increment $x_{i}^{\prime}(n)(i \geq 2)$. The strain and the corresponding stretching factor were determined based on the pre-specified computer controlled step motor movement. At very small strains, the correlation coefficients in Fig. 2 are similar to each other with or without stretching. The correlation coefficient for the un-stretched case represented by the dashed line decreases rapidly to below 0.1 as the strain increases. On the other hand, the correlation coefficient with stretching declines relatively slowly. At a $2 \%$ strain, the correlation coefficient drops to slightly below 0.2 . In other words, although temporal stretching has been applied, significant decorrelation is still present. Therefore, reduction of speckle fluctuations by compounding is feasible.

The speckle signal-to-noise ratio (SNR), defined as the ratio of mean to standard deviation of the amplitude of the detected signal, is used to represent efficacy of speckle reduction. For fully developed speckle, Rayleigh probability density function can be applied. In this case, the speckle SNR approximates 1.91. The speckle SNR of the compound signal under different strain conditions is shown in Fig. 3 . The dashed line represents the SNR without stretching and the solid line denotes the SNR with stretching. The correlation decreases as the strain increases. Thus, speckle fluctuations after incoherent averaging decrease and contrast resolution is improved.

Fig. 3 indicates that with a $2 \%$ strain (i.e., about $1 \mathrm{~mm}$ deformation), the speckle brightness variations can be reduced by roughly $20 \%$ by using only two measurements.

\section{COMPOUNDING}

To further reduce speckle fluctuations, multiple measurements with a larger applied strain must be employed. Fig. 4 shows correlation coefficients obtained by compressing another phantom with an increment of $150 \mu \mathrm{m}$. The phantom was 50 $\mathrm{mm}$ high with a hyperechoic cylindrical inclusion. There were totally 20 compression steps corresponding to a total strain of $5.7 \%$. The mean brightness of the inclusion was about $5.5 \mathrm{~dB}$ higher than that of the background. Results in Fig. 4 show that the correlation coefficient reduces to a minimum at a strain near $1.2 \%$ (i.e., 4 compression increments). In other words, no further reduction can be gained with a step size larger than 4 if only two measurements 
are available. Therefore, compounding can be done by using one out of every 4 increments (i.e., measurements $1,5,9,13$ and 17) and speckle variations are expected to reduce as the number of measurements increases.

The resulting images are shown in Fig. 5 with a display dynamic range of $30 \mathrm{~dB}$. Starting from the top left corner, the images correspond to the original image (measurement 1), the compound image using measurements 1 and 5 (top right), the compound image using measurements 1,5 and 9 (bottom left), and the compound image using measurements $1,5,9$ and 13 (bottom right), respectively. It is shown that speckle brightness variations are more effectively reduced as the number of measurements increases. As the speckle variations are reduced, both contrast resolution and edge definition of the inclusion are improved.

Fig. 6 shows improvement in contrast resolution as a function of number of measurements used for compounding. A contrast-to-noise ratio (CNR) was used as a quantitative measure. All values in this figure are normalized to the CNR of the original image. The un-normalized CNR is defined as the following:

$$
C N R \equiv \frac{\left|m_{t n}-m_{o u t}\right|}{\sigma_{\text {in }}+\sigma_{\text {out }}},
$$

where $m_{m}$ and $m_{o u t}$ are mean brightness values inside and outside of the inclusion, respectively, and $\sigma_{i n}$ and $\sigma_{i u t}$ are the standard deviation values inside and outside of the inclusion, respectively. All values were calculated based on the detected images prior to logarithmic conversion. The solid line in Fig. 6 is the CNR using experimental results. The dashed line is an ideal curve assuming all measurements are completely un-correlated. In this case, the ideal curve follows the relation normalized $C N R=\sqrt{N}$, where $N$ is the number of un-correlated measurements. With 5 measurements, the CNR was increased by more than two times at an applied strain of $4.8 \%$.

\section{CONCLUSIONS}

A new compounding technique was proposed. Since incoherent averaging is done on images under different strain states, the technique was also referred to as strain compounding. It was shown that by applying a strain as small as $4.8 \%$, speckle fluctuations can be reduced effectively and the CNR can be more than doubled. Such an improvement may be further increased at the same applied strain if optimal compression strategies can be found.

\section{REFERENCES}

[1] P. A. Magnin, O. T. von Ramm, and F. L. Thurstone, "Frequency compounding for speckle contrast reduction in phased array images," Ultrason Imaging, vol. 4, pp. 267-281, 1982.

[2] D. P. Shattuck and O. T. von Ramm, "Compound scanning with a phased array," Ultrason Imaging, vol. 4, pp. 93-107, 1982.

[3] M. O'Donnell and S. D. Silverstein, "Optimum displacement for compound image generation in medical ultrasound," IEEE Trans. Ultrason., Ferroelect., Freq. Contr. vol. 35, pp. 470-476, 1988.

[4] P.-C. Li and M. O'Donnell, "Elevational spatial compounding," Ultrason Imaging, vol. 16, pp. 176-189, 1994.

[5] M. O'Donnell, A. R. Skovoroda, B. M. Shapo, and S. Y. Emelianov, "Internal displacement and strain imaging using ultrasonic speckle tracking," IEEE Trans. Ultrason., Ferroelect, Freq. Contr., vol. 41, pp. 314-325, 1994.

[6] T. Varghese and J. Ophir, "Enhancement of echo-signal correlation in elastography using temporal stretching," IEEE Trans. Ultrason., Ferroelect., Freq. Contr., vol. 44, pp. 173-180, 1997. 


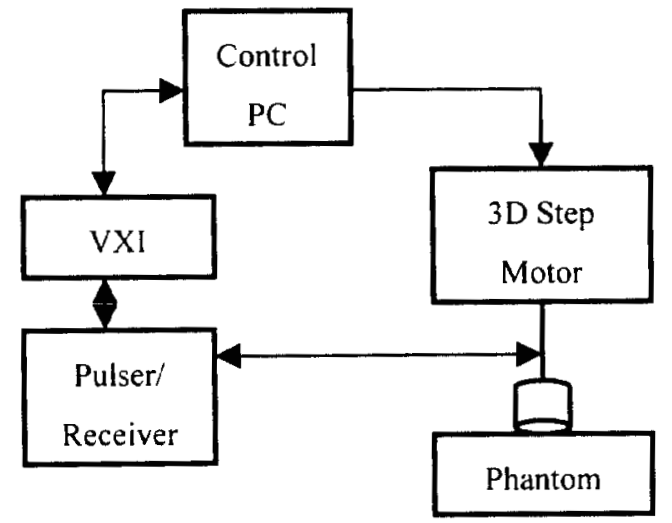

Fig. 1: Experimental Setup.

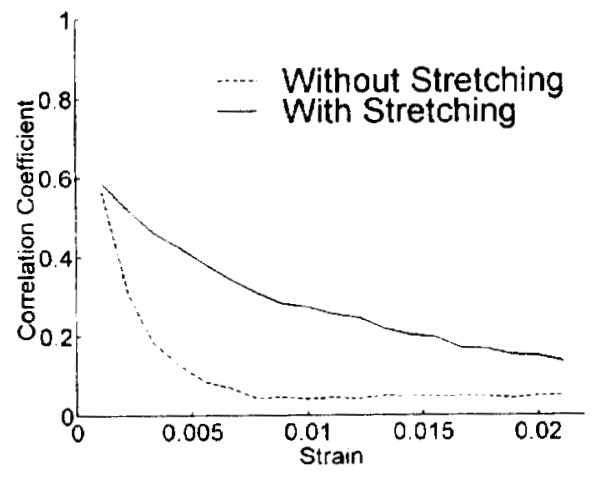

Fig. 2: Correlation Coefficients.

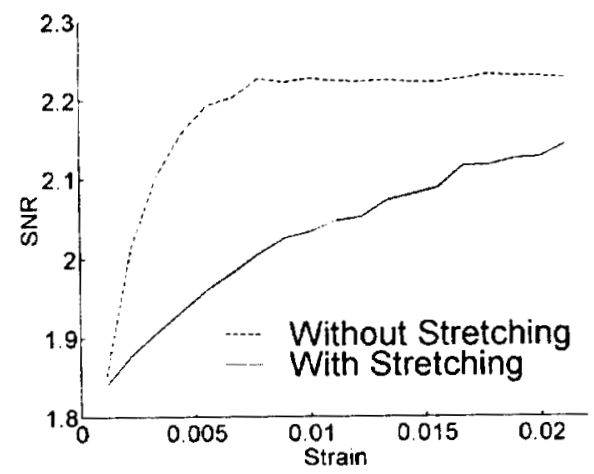

Fig. 3: Speckle SNR.

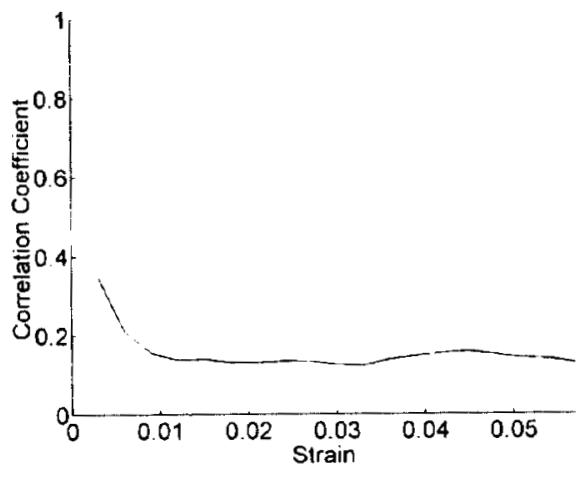

Fig. 4: Correlation Coefficients.
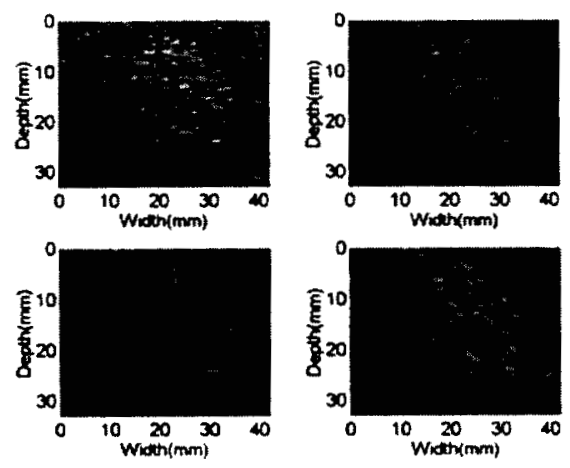

Fig. 5: Compound Images.

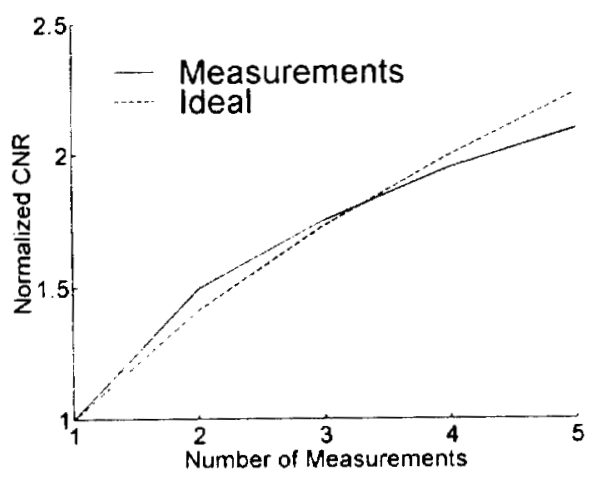

Fig. 6: Normalized CNR. 